\title{
Stratégie de gestion de l'irrigation en conditions de ressources limitées
}

\author{
J. Puech*, F. Levrault*, N. Bosc*, J.-P. Amigues** \\ I.N.R.A. - Centre de Recherches de Toulouse \\ * Station d'Agronomie - Tél. : 61285033 - Fax: 61735537 \\ ** Laboratoire d'Economie et Sociologie Rurales - Tél. : 61205093 - Fax : 61732077 \\ BP 27 - F 31326 Castanet Tolosan Cedex
}

\begin{abstract}
Dans le contexte actuel de rareté relative des ressources en eau et de risques de pollution des nappes, il paraît très important de bien maîtriser la gestion de l'eau. Cette démarche peut se concevoir tant par des effets de protection de l'environnement que par un souci de répartition de ressources limitées à valoriser, techniquement, au mieux. Ainsi, dans le domaine agricole, les sécheresses de ces dernières années en France ont amené les pouvoirs publics, à mettre en œuvre des cellules "sécheresse" au niveau départemental et régional (notamment dans la région Midi-Pyrénées). Cette procédure a permis, à plusieurs reprises, de dégager les critères à retenir pour s'adapter à des restrictions en eau tant en volume total qu'en moment d'accession autorisée à la ressource et à atténuer les conflits d'usage entre les acteurs économiques. Du point de vue agronomique, il s'est donc agi de modifier à temps les assolements pratiqués (recherche d'espèces et d'assolements économiseurs d'eau), d'effectuer des changements de dates de semis, de groupe de précocité, de répartir différemment l'eau disponible avec les espèces en concurrence, en évaluant les risques compte tenu des conditions pédoclimatiques. De ces expériences, il ressort la nécessité de bien cerner le fonctionnement hydraulique de bassins versants plus ou moins complexes, et le fonctionnement hydrique des divers systèmes de culture pratiqués ou adaptés, en vue de mettre en œuvre des solutions de substitution aux scénarios antérieurs. Cette approche implique, du point de vue agronomique, le choix d'assolements viables les mieux adaptés à ce type de contrainte.
\end{abstract}

\section{Stratégies du choix des assolements}

Les conditions imposées par la moindre disponibilité des ressources hydriques, amènent à concevoir des choix stratégiques pour les irrigants. Or, la gestion de l'eau ne peut se raisonner qu'en considérant le fonctionnement global de l'exploitation agricole, en tenant compte de facteurs techniques (disponibilités de ressources en eau, matériel, choix des espèces...) économiques (prix de commercialisation) et réglementaires (règles $\mathrm{CEE}$ ).

Pour bien assurer ces choix, divers logiciels d'aide à la décision basés sur des modèles informatiques performants permettent de réaliser des choix d'assolement par rapport à des contraintes définies.

Par exemple, le logiciel GEDE (BEYRIES, 1990), basé sur la programmation linéaire, permet de simuler une stratégie globale de l'exploitation, en explicitant diverses interactions et en précisant les opportunités d'équipement, de valorisation d'une ressource (etc...).

Dans d'autres cas, le logiciel LORA (LEROY, 1991), recherche un assolement maximisant la marge brute totale, techniquement compatible avec la ressource en eau, l'équipement, la main-d'œuvre, la variabilité climatique, etc...

Les simulations ainsi réalisées permettent d'affiner les choix d'assolement, d'évaluer les marges de manœuvre, de préciser les ajustements nécessaires de surfaces à irriguer et selon quelles modalités.

Dans ces conditions, si sur une exploitation on a réussi à optimiser les choix d'ordre stratégique, fixant le système de culture à retenir, il reste à adapter les choix tactiques, en temps quasi réel, compte tenu des conditions climatiques, des évolutions phénologiques, des surfaces en jeu et de leur niveau d'hétérogénéité, des contraintes de matériel et de débit. Parmi les solutions classiquement retenues pour arrêter les choix tactiques, il est souvent fait appel à des mesures "ponctuelles " type tensiomètre, humidimétrie, etc... supposées représenter au mieux, à partir de mesures sur des surfaces limitées, l'ensemble de la ou des parcelles d'une culture donnée.

Or, en matière de choix tactique, on peut concevoir aussi la mise en œuvre de modèles de simulation permettant de représenter divers scénarios d'évolution de la culture, compte tenu des choix faits, tout en incluant divers critères de variation.

Cette approche permet, alors, d'analyser le fonctionnement de rotations culturales avec différentes conditions pédoclimatiques et niveaux d'intrants, d'où une évaluation des risques possibles. 


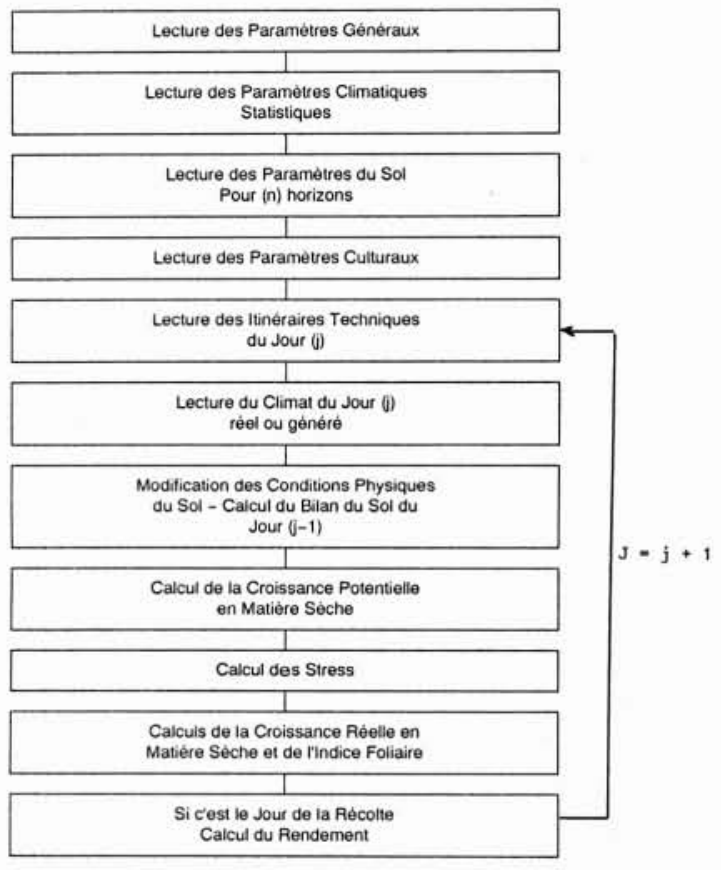

1 Organigramme du modèle EPIC.

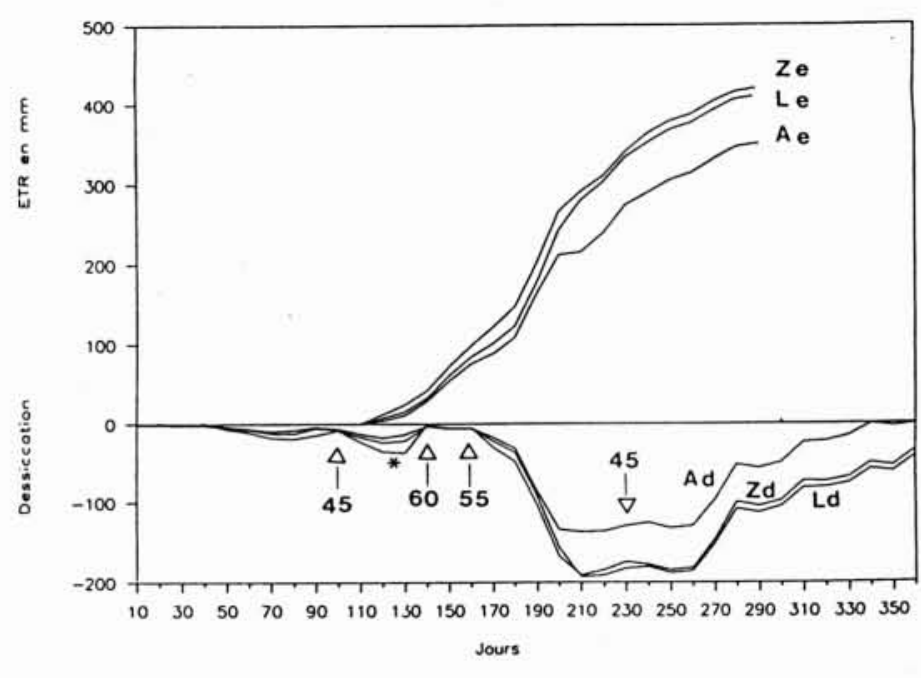

4 Comparaison de la dessiccation du sol et de la consommation d'eau par une culture de maïs sur 3 types de sols argileux (Ae). limoneux (Le) et limono-argileux ( $\mathrm{Ze}$ ) au cours d'une année (sol de profondeur moyenne $1,1 \mathrm{~m}$ ). Pour les ETR seule la période de culture est mentionnée.

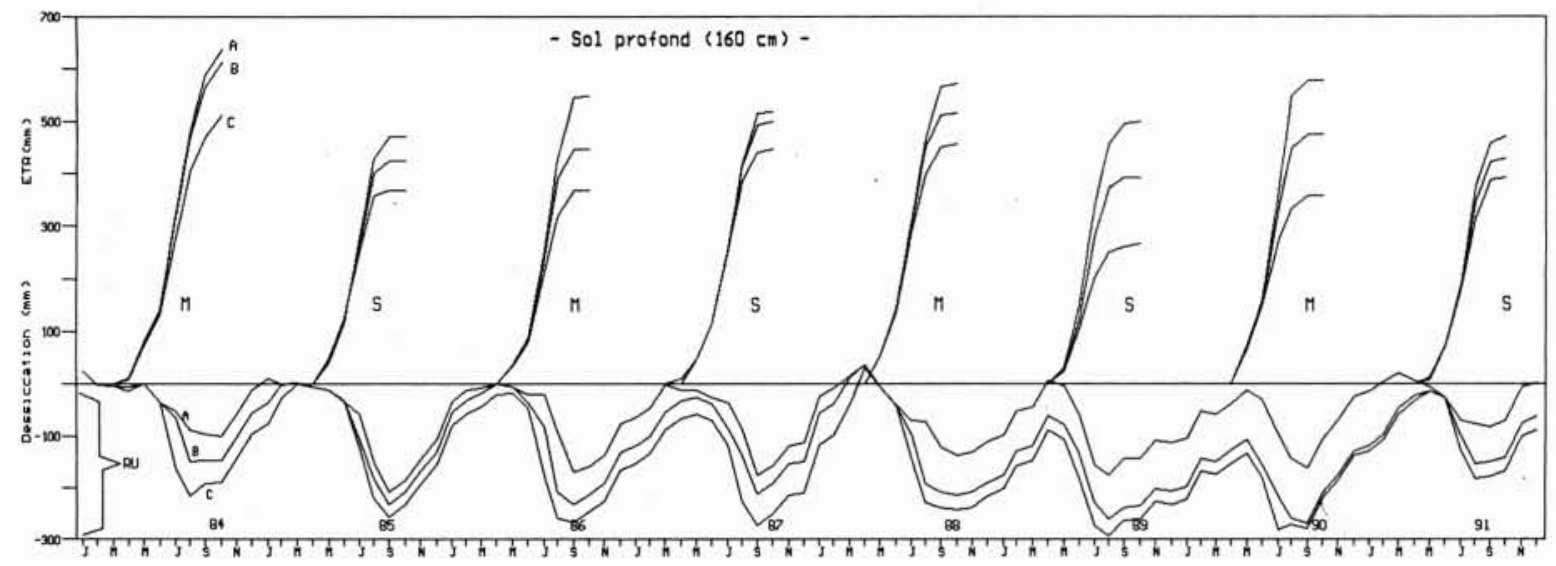

2 Evolution au cours de 8 années de l'évapotranspiration réelle $(\mathrm{mm})$ et de la dessiccation $(\mathrm{mm})$ d'un sol profond $(1,60 \mathrm{~m})$ par une rotation maïs-soja menée avec différents niveaux $(A-B-C)$. Les valeurs d'évaporation en période de non culture ne sont pas reportées.

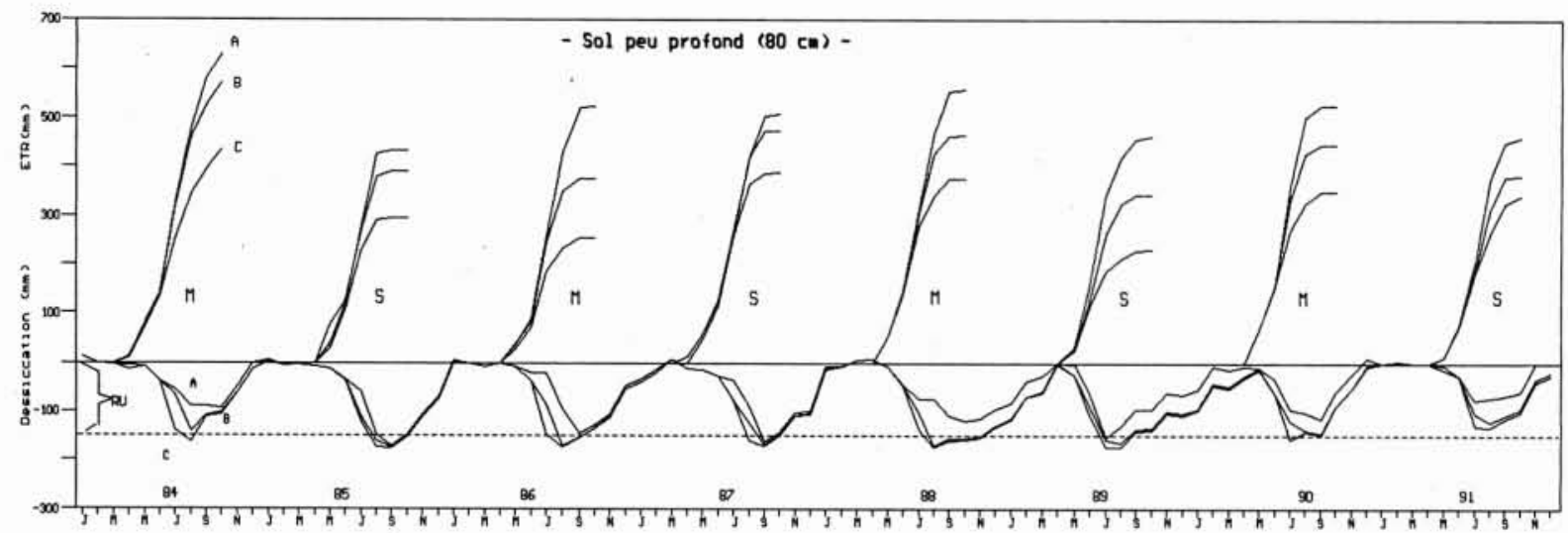

3 Evaluation au cours de 8 années de l'évapotranspiration réelle $(\mathrm{mm})$ et de la dessiccation $(\mathrm{mm})$ d'un sol peu profond $(0,80 \mathrm{~m})$ pour une rotation maïs-soja menée avec différents niveaux d'intrants $(A-B-C)$. Les valeurs d'évaporation en période de non-culture ne sont pas reportées. 


\section{Matériel et méthodes}

Dans cette étude, nous avons utilisé le modèle EPIC (CABelguenne et al., 1990), modèle photosynthétique à pas de temps journalier, à large specte exploratoire (fig. I). Il prend en compte les relations entre la plante, le sol et le climat, ainsi que les intrants et les itinéraires techniques à partir de paramètres culturaux élaborés pour plusicurs espèces. Le modèle peut simuler, à l'aide d'un générateur de climats, plusieurs scénarios météorologiques.

Les données ont été calibrées et validées à partir des données du dispositif expérimental de la Station d'Agronomie de Toulouse-Auzeville concernant le fonctionnement de differrents systemes de cultures en fonction de divers niveaux d'intrants.

Le modèle traite de situations "parcellaires " considérées comme isotropes en ce qui concerne le sol retenu (type et caractéristiques, données, profondeur...). Aussi, pour intégrer la composante spatiale prenant en compte le rôle et l'importance des hétérogénéités au sein d'une parcelle, nous nous sommes appuyés sur des données de téléobservation et des résultats d'analyse numérique d'images en infrarouge couleur (logiciel CARTO-PC, Ecole des Mines de Paris) sur des cultures de maïs et de soja. Ces données permettent de définir des paramètres correcteurs quant aux surfaces à comportement différencié au sein d'une parcelle donnée.

\section{Comportement hydrique de rotations culturales types}

Une fois l'assolement déterminé, il est important d'analyser le fonctionnement de succession de cultures types pour bien dégager les cinétiques hydriques et en corollaire, l'évolution de la biomasse produite. Des travaux antérieurs (PUECH et al., 1978) nous avaient permis de mettre en évidence le fonctionnement au champ de rotations culturales types. Aussi, nous dégagerons ci-après, les informations importantes, à partir de simulations effectuées avec le modèle EPIC de façon à prendre en compte des scénarios plus complexes. La rotation maïs-soja du dispositif d'Auzeville, menée selon 3 niveaux d'intrants (culture en sec - C -, culture modérément irriguée - B -, et culture menée sans facteurs limitants - A -) servira de rotation type.

Le tableau $I$ montre les productions atteintes et la figure 2 montre le fonctionnement hydrique de ce système dans le cas d'un sol profond $(1,60 \mathrm{~m})$ et pour 8 années consécutives 84-91. En ce qui concerne l'évapotranspiration réelle (ETR) de la culture on note une différence importante chaque année entre les niveaux d'intrants pratiqués et des variations plus ou moins grandes d'utilisation des réserves du sol. En particulier, il apparaît les périodes de dessiccation maximale, les époques de réhumectation, les durées de capacité au champ et les risques de lessivage correspondants selon le niveau d'intensification pratiqué. Par ailleurs les courbes $\mathrm{A}$ et $\mathrm{C}$ présentent les situations hydriques extrêmes (en conditions non limitantes et sèches), l'écart entre ces courbes définissant la marge de manœuvre permise chaque année pour atteindre divers objectifs intermédiaires.
Tableau 1. Production simulée ( $q / h a)$ sur 4 années différentes pour le maïs et le soja en fonction de 3 niveaux d'intrants $A$ en sec, $B$ irrigation modérée, C irrigation forte. Cas d'un sol profond.

\begin{tabular}{|c|c|c|c|c|c|c|c|c|c|}
\hline \multirow{2}{*}{$\begin{array}{l}\text { Années } \\
\begin{array}{c}\text { MAIS } \\
1984\end{array}\end{array}$} & \multicolumn{3}{|c|}{ 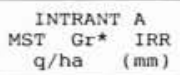 } & $\begin{array}{r}\text { MST } \\
\text { q } /\end{array}$ & $\begin{array}{l}\text { TRAN } \\
\text { Gr* } \\
a^{\star}\end{array}$ & $\begin{array}{l}{ }_{\text {IRR }} \\
(\mathrm{mm})\end{array}$ & $\begin{array}{r}\text { MST } \\
\mathrm{q}\end{array}$ & $\begin{array}{l}\text { RANT } \\
\text { Gr* }\end{array}$ & $\begin{array}{l}c_{\text {IRF }} \\
(\mathrm{mm})\end{array}$ \\
\hline & 214 & 77 & 0 & 224 & 120 & 155 & 251 & 125 & 225 \\
\hline 1986 & 190 & 51 & 0 & 226 & 103 & 110 & 257 & 127 & 280 \\
\hline 1988 & 213 & 77 & 0 & 238 & 110 & 90 & 245 & 122 & 240 \\
\hline 1990 & 138 & 25 & 0 & 188 & 69 & 100 & 215 & 102 & 235 \\
\hline $\begin{array}{l}\text { SOJA } \\
1985\end{array}$ & 98 & 28 & 0 & 104 & 31 & 80 & 111 & 32 & 155 \\
\hline 1987 & 102 & 31 & 0 & 105 & 32 & 80 & 105 & 32 & 130 \\
\hline 1989 & 69 & 17 & 0 & 92 & 28 & 120 & 106 & 32 & 280 \\
\hline 1991 & 84 & 25 & 0 & 89 & 27 & 60 & 95 & 29 & 185 \\
\hline
\end{tabular}

Ce type de résultats, avec des amplitudes plus ou moins grandes, se retrouve si l'on considère des fluctuations de la profondeur de sol. La figure 3 montre les résultats obtenus avec les mêmes niveaux d'intrants que précédemment mais avec un sol de profondeur moindre $(0,80 \mathrm{~m})$. Il apparaît une nette fluctuation des niveaux d'ETR atteints et des niveaux de dessiccation de sol. Dans le cas de cultures en sec, la réserve utile, entamée très tôt, est pratiquement épuisée chaque année. En culture irriguée, avec les intrants pratiqués, on constate que les dessiccations peuvent être très fortes, même en scénario $(\mathrm{A})$, correspondant à une alimentation non limitante en eau dans le cas du sol profond.

Enfin, si en plus des effets des variations de profondeur on fait intervenir une modification des caractéristiques texturales, on met alors en évidence des effets dus aux cessibilités en eau différentes. La figure 4 montre les résultats obtenus avec 3 types de sol, en considérant des conditions initiales identiques (même intrant, sol, initialement à la capacité au champ). Il apparaît notamment la moindre participation des réserves hydriques en sol argileux par rapport aux sols plus limoneux et une ETR inférieure.

De cet ensemble de données, on peut tirer les indications suivantes :

1) $\mathrm{Si}$ on se fixe un niveau donné de production, il faut pouvoir, pour atteindre cet objectif, simuler le rythme de fonctionnement de la culture selon les conditions pédoclimatiques du lieu et notamment pouvoir tenir compte des hétérogénéités parcellaires d'ordre divers ;

2) L'analyse des courbes enveloppes obtenues (en ETR ou MST par exemple), montre qu'il existe un point de divergence entre les courbes en sec et celles en eau non limitante à des moments variables selon les années. Les simulations permettent de définir :

- d'une part la durée pendant laquelle il y a identité de comportement (soit entre les niveaux d'intrants pratiqués, soit entre les sols différents) donc ne nécessitant aucune intervention corrective ;

- d'autre part de préciser le moment où une divergence s'installe. 

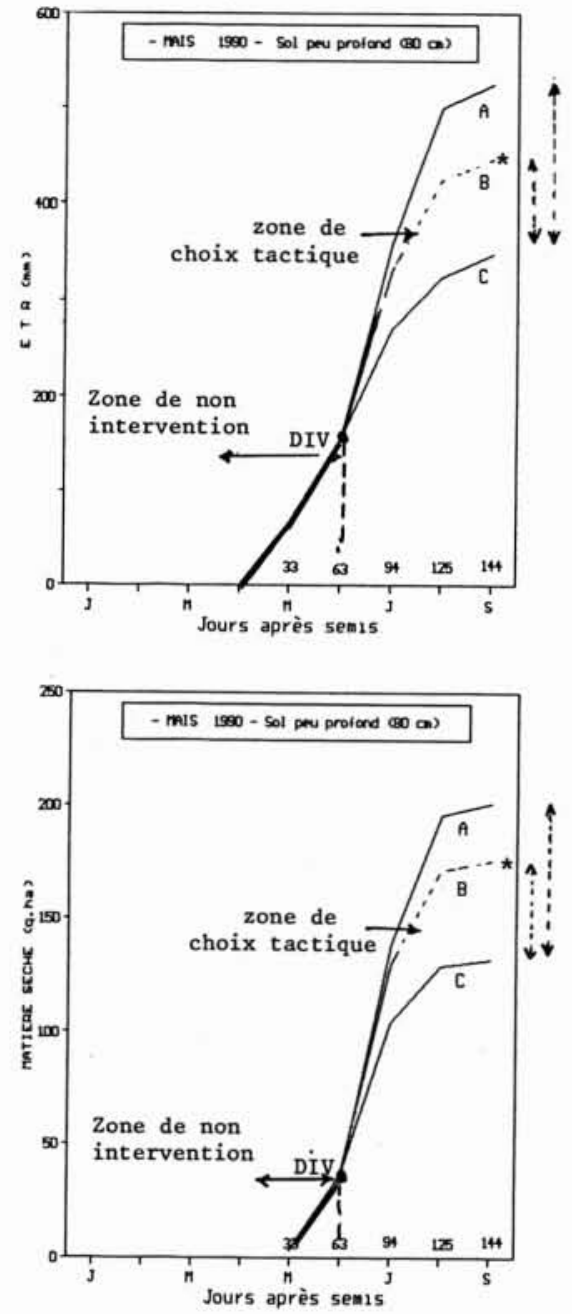

* objectif à atteindre.

5 Importance du point de divergence entre des simulations en condition sèche $(C)$ et en eau non limitante $(A)$. Itinéraire probable pour un objectif intermédiaire (B) (ETR ou MST).

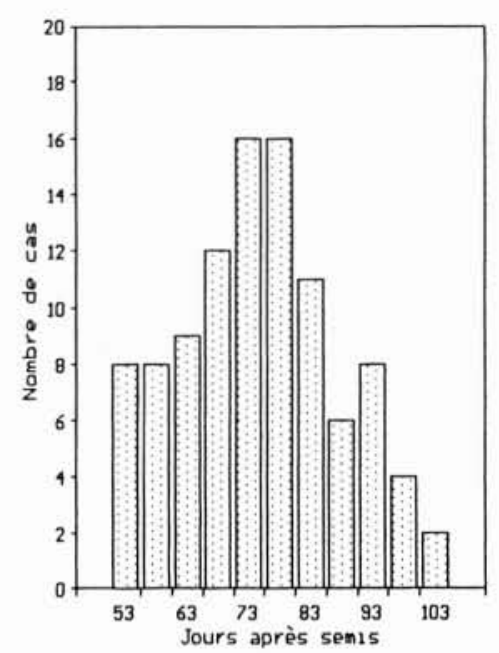

6 Nombre de cas d'apparition en fonction du nombre de jours après semis, du point de divergence : simulation sur maïs sur 100 ans avec le générateur de climat.

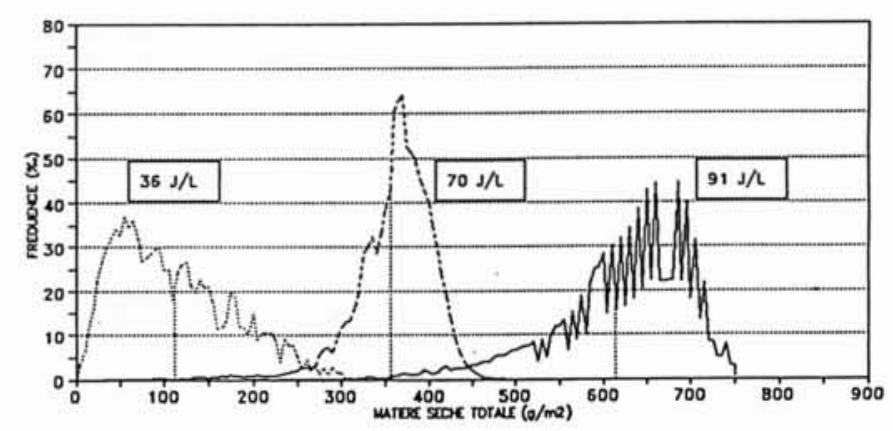

7 Evolution au cours du temps de la distribution statistique des matières sèches totales sur une parcelle de soja irriguée de manière homogène (estimation à partir de l'imagerie numérique en IRC). Les lignes pointillées verticales représentent la moyenne parcellaire observée à chaque date.

Ce schéma se complique, si au lieu d'un sol isotrope, on a affaire à un sol hétérogène (en profondeur, en texture, etc...). Il s'agit alors de pouvoir simuler le comportement par zones équivalentes et d'en déduire ensuite une simulation moyenne représentative du système.

Dans tous les cas, on est ramené à la définition de la zone du point de divergence (fig. 5) et à l'établissement de la "valeur d'écart entre les courbes enveloppes" qui déclenche, à valeur seuil, une intervention compte tenu des ressources disponibles. Il s'agit donc d'évaluer les risques courus concernant, d'une part la durée entre le point de divergence et la maturité et d'autre part, l'occurrence de pluie.

A titre d'illustration, la figure 6 indique, pour une simulation sur 100 années l'apparition du point de divergence entre une culture de maïs en sec et en eau non limitante; il existe une variabilité importante avec apparition du point de divergence entre le $53^{\mathrm{e}}$ et le $103^{\mathrm{e}}$ jour avec une plus grande fréquence vers $70-80$ jours après semis. Les divergences sont très précoces en année sèche et au contraire tardives en année faiblement évaporante. Des études complémentaires, relatives aux paramètres prédicteurs de ces points sont donc à entreprendre ; en effet, les points de divergence sont prédicteurs des comportements ultérieurs des cultures, ils précisent la « mise en route » d'un scénario pour un rendement donné (culture en sec par exemple). Le contrôle de l'apparition du point de divergence pourrait être une méthode d'évaluation des risques et des possibilités pour atteindre un rendement donné avec des contraintes fixées. La procédure nécessite des hypothèses sur le devenir climatique proche, qui peut être soit abordé par des simulations à partir du générateur de climat du modèle, soit par une adaptation des prévisions à 5 jours de la Météorologie Nationale, avec ajustement glissant dans le temps. 
On peut aussi concevoir la création de deux courbes " guides " servant d'enveloppes (l'une caractérisant une situation en sec, l'autre en eau non limitante) encadrant les données réelles de l'année testée. Enfin, les simulations peuvent être effectuées avec différents régimes d'apport d'eau pour voir la répartition possible en cas de sécheresse ou de pluies compensatrices.

La simulation du comportement de plusieurs parcelles et de plusieurs scénarios sur cultures peut aider à faire des impasses d'intervention sur certaines zones, à anticiper ou même à privilégier une culture plutôt qu'une autre compte tenu de la configuration des conditions de l'année. Ces déductions sont en accord avec ce qui peut être observé du comportement hydrique d'une parcelle à partir de moyens de téléobservation. En effet, si on procède à une analyse numérique d'image à partir de clichés obtenus en infrarouge couleur ou en infrarouge thermique (LEVRAULT, 1992 ; LeVrault et al., 1992), on met en évidence différentes zones de comportement hydrique sur une parcelle donnée menée selon une procédure agronomique unique. Si par ailleurs, sur les images numériques on établit la distribution des matières sèches par unité de surface détectée, on observe par exemple (fig. 7) l'importance des dysfonctionnements au cours du temps et à l'intérieur de la parcelle. Cela confirme la démarche précédente déduite du modèle EPIC, où la mise en évidence des fluctuations des points de divergence sur des simulations avec divers paramètres d'entrée (profondeur de sol, texture, etc...) indique des fonctionnements différents intraparcellaires.

\section{Conclusion}

L'étude du fonctionnement hydrique d'un système de culture défini à partir de considérations économiques et agronomiques optimisées amène plusieurs observations :

- pour obtenir une gestion performante il faut pouvoir définir la quantité minimale d'eau effectivement accessible et sa disponibilité dans le temps (périodes connues ou aléatoires);

- par ailleurs, plutôt que de recourir uniquement aux techniques habituellement utilisées pour définir l'état hydrique des cultures, techniques plus ou moins ponctuelles, nous proposons également la mise en œuvre de modèles permettant l'étude cinétique du fonctionnement hydrique de la culture. Cette approche autorise l'essai de plusieurs scénarios combinant des fluctuations de sol (caractéristiques texturales, hydriques, chimiques ; profondeur, etc...) de climat (combinaison de scénarios plus ou moins contraignants) et de cultures : substitution d'espèces pour voir les répercussions sur les bilans d'eau et sur la biomasse produite. Cette démarche permet alors de tester plusieurs combinaisons de cultures avec différents types d'itinéraires techniques (notamment d'apport d'eau); elle amène à retenir les successions de culture présentant les arrière-effets les moins défavorables.

Enfin, la connaissance de l'existence d'un point de divergence entre une situation dite sans facteur limitant et une situation en sec permet d'évaluer les niveaux de risques en jouant sur l'état des réserves, des ressources, du climat à partir de simulations tests.

\section{Bibliographie}

Beyries Ph. (1990). - Gestion de l'eau dans les exploitations de grande culture du Sud-Ouest de la France: pratiques et problèmes. CEMAGREF - Groupement de Bordeaux, $63 \mathrm{p}$.

Leroy P., JACQuin C. (1991). - « LORA a decision support system for choice of crops on the irrigable area of a farm ". Congrès "Decision support System I.F.O.R.S.-S.P.C.I., Bruges, mars 1991.

Cabelguenne M., Jones C.A., Marty J.-R., Dyke I.T., WiLliams J.-R. (1990). - Calibration and validation of EPIC for crop rotations in Southern France. Agricultural Systems, 33, 153-171.

LeVrault F. (1992). - Analyse par imagerie IRC du fonctionnement de parcelles de soja plus ou moins irriguées: appli- cation à la gestion de l'eau dans les systèmes de cultures. Thèse Université P. Sabatier, Toulouse. INRA-CETIOM, $229 \mathrm{p}$.

Levrault F., Puech J. (1992). - Gestion de l'eau, sur un système diversement intensifié, par imagerie infrarouge couleurs: approche expérimentale sur une parcelle de soja. Agronomie (en cours d'impression).

Levrault F., Puech J., Yacouba H. (1992). Modélisation spatialisée de la croissance, de parcelles de soja plus ou moins irriguées. Conférence : Plant Water Relations under water stress environments. Bari 27/9-21/10/92.

Puech J., Marty J.-R., Hernandez M. (1978). Bilans hydriques pluriannuels sous diverses rotations culturales irriguées ou non. In : l'Hydrotechnique au service d'une politique de l'eau. $X V^{\text {es }}$ Journées de l'Hydraulique - SHF - Question IV rapport 4 (1-6). 


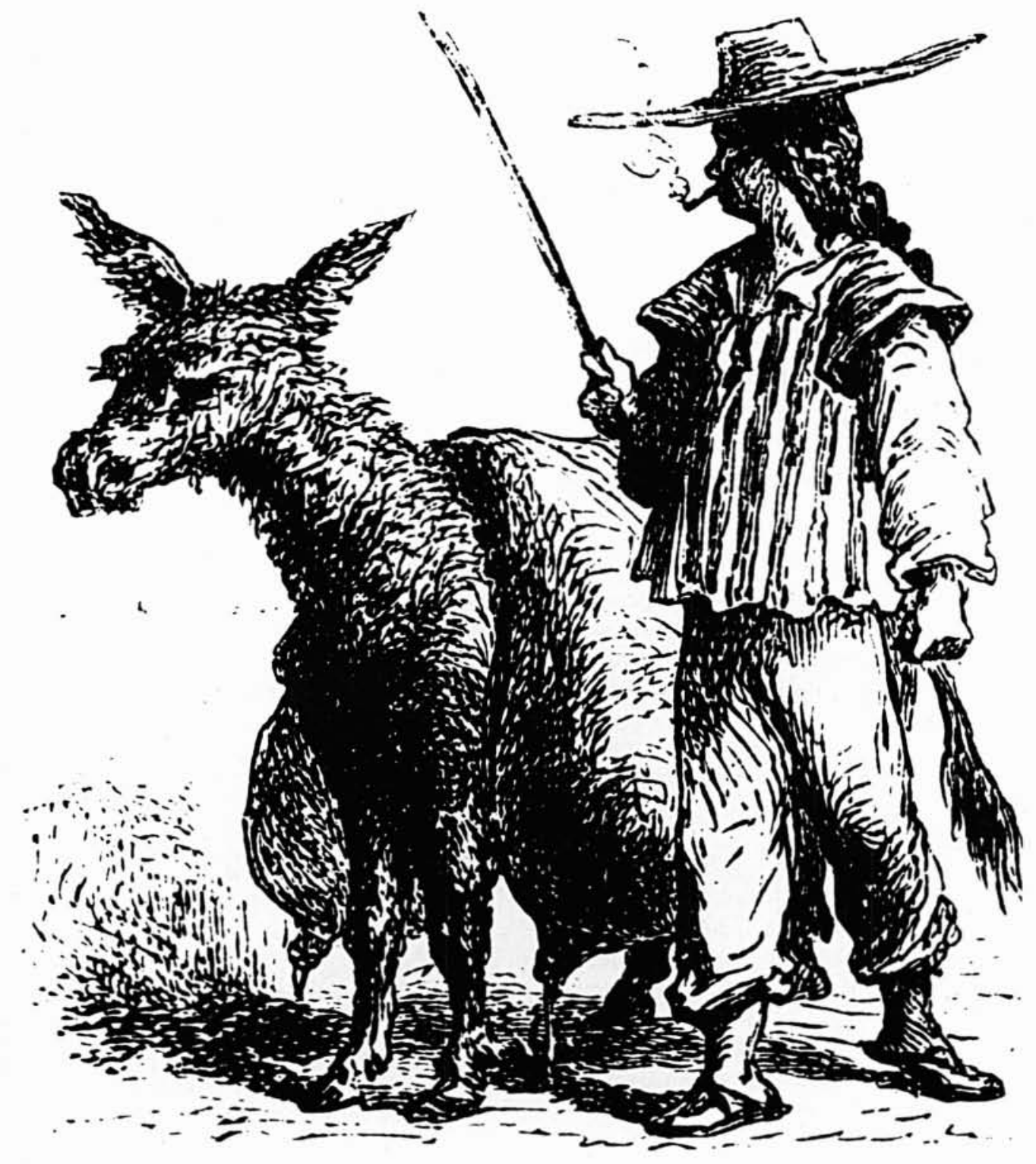

